\title{
Liame
}

Histoire et histoire de l'art des époques moderne et contemporaine de l'Europe méditerranéenne et de ses

périphéries

\section{Collections antiquaires et identité méridionale.Les travaux du nîmois Anne de Rulman (1582-1632)}

\section{Marianne Freyssinet}

\section{(2) OpenEdition}

\section{Journals}

Electronic version

URL: http://journals.openedition.org/liame/567

DOI: $10.4000 /$ liame.567

ISSN: 2264-623X

\section{Publisher}

CRISES - Centre de Recherches Interdisciplinaires en Sciences Humaines et Sociales de Montpellier

\section{Electronic reference}

Marianne Freyssinet, «Collections antiquaires et identité méridionale.Les travaux du nîmois Anne de Rulman (1582-1632) », Liame [Online], 26 | 2016, Online since 14 March 2016, connection on 19 April 2019. URL : http://journals.openedition.org/liame/567 ; DOI : 10.4000/liame.567

This text was automatically generated on 19 April 2019

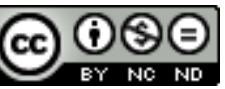

Les contenus de Liame sont mis à disposition selon les termes de la Licence Creative Commons Attribution - Pas d'Utilisation Commerciale - Pas de Modification 4.0 International. 


\title{
Collections antiquaires et identité méridionale.Les travaux du nîmois Anne de Rulman (1582-1632)
}

\author{
Marianne Freyssinet
}

1 Dans son article paru dans le Journal of the Warburg and Courtauld Institutes en 1950, Arnaldo Momigliano définit l'antiquaire comme « celui qui aime, rassemble et étudie les traditions et les restes du monde antique, sans être historien ${ }^{1}$ » ajoutant encore que cette notion est l'une des plus caractéristiques de l'humanisme des $\mathrm{XV}^{\mathrm{e}}$ et $\mathrm{XVI}^{\mathrm{e}}$ siècles ${ }^{2}$ ». Outre l'élaboration d'une méthode historique nouvelle, Momigliano mentionne la révolution du goût qui marque l'ère des antiquaires: "L'antiquaire était un connaisseur et un enthousiaste; il habitait un monde statique, il avait pour idéal de collectionner ${ }^{3} »$. On comprend alors le lien étroit tissé entre d'un côté l'élaboration d'une science antiquaire fondée sur le rassemblement et l'étude des antiques, et de l'autre, les «curieux d'histoire " pour reprendre l'appellation d'Antoine Schnapper ${ }^{4}$. Ce dernier note à cette occasion la place de choix qu'occupent les antiquités au sein des collections de Provence et du Languedoc, du fait notamment des découvertes archéologiques dans la France méridionale du Grand Siècle, qui «alimentent les cabinets des curieux comme leurs disputes $^{5} \%$. Un constat partagé quelques décennies plus tard par Véronique Krings, qui consacre une étude aux antiquaires du Midi en s'attachant à une large période - allant du $\mathrm{XVI}^{\mathrm{e}}$ au XIX ${ }^{\mathrm{e}}$ siècle - et décrit à son tour, un « milieu particulièrement riche ${ }^{6}$ ».

2 De fait, le Midi de la France constitue un terrain d'études fécond pour l'appréhension de la curiosité antiquaire à l'époque moderne, ne serait-ce que par son prestigieux passé antique, illustré par la présence de nombreux vestiges monumentaux. Au XVII ${ }^{e}$ siècle, Nîmes disposait ainsi avec la Maison Carrée, les Arènes et le pseudo-temple de Diane de monuments majeurs. Associé au pont du Gard, son ensemble monumental faisait partie des plus importants sites issus du monde romain. Venait ensuite Arles qui avait elle aussi été dotée d'un riche programme urbanistique comprenant amphithéâtre, théâtre, forum, arcs et cirque et passait alors pour une seconde Rome ${ }^{7}$. S'ajoutaient encore Vienne et son 
Temple d'Auguste et de Livie, Orange et son arc de triomphe : autant de sites qui pouvaient s'enorgueillir de posséder des traces visibles de leur passé antique et suscitaient l'intérêt des amateurs ${ }^{8}$. À cet égard, le tournant entre le XVI ${ }^{\mathrm{e}}$ et le XVII ${ }^{\mathrm{e}}$ siècle marque un temps fort avec des personnalités emblématiques telles que Nicolas-Claude Fabri de Peiresc (1580-1637) autour duquel gravitent collectionneurs provençaux et, au-delà, européens. Une génération qui, outre ses collectionneurs, a également laissé de nombreuses descriptions des antiquités du Sud de la France.

3 C'est au sein de ce milieu des antiquaires du Midi et de cette génération au tournant du siècle qu'il convient de situer Anne de Rulman (1582-1632). Né à Nîmes en 1582, cet avocat a laissé à la suite d'une commande royale, un ensemble de manuscrits de grande ampleur aujourd'hui répartis entre la Bibliothèque nationale de France et la Bibliothèque du Carré d'Art de Nîmes'. Cet ensemble, écrit entre 1626 et 1630, est consacré aux antiquités des territoires de l'ancienne Gaule Narbonnaise ; il comprend plusieurs parties inachevées et inclut également un volume de dessins d'antiquités essentiellement nîmoises, mais portant en partie aussi sur Béziers ${ }^{10}$. Une source qui permet ainsi d'interroger la place des antiquités et de l'Antiquité au sein des milieux de curieux méridionaux et de mesurer si et si oui, en quoi ? - la proximité des vestiges a pu engendrer une spécificité des pratiques, aussi bien de la collection, que des discours. Plus encore, a-t-elle donné lieu à l'apparition d'une élite antiquaire à l'identité caractérisée?

\section{Le sol du Midi et les trouvailles antiques}

4 Le premier constat qui s'impose à la lecture du texte de Rulman repose sur le nombre très important de trouvailles d'antiquités dont il fait état, un nombre qui renforce l'idée de découvertes incessantes effectuées dans la France méridionale du Grand Siècle. Tout au long de l'époque moderne, le sol du Midi prodigue en effet de nombreux vestiges découverts, pour la plupart, lors des travaux des champs ou travaux des villes, particulièrement fréquents au XVII ${ }^{\mathrm{e}}$ siècle comme le rappelle Pierre Pinon, avec les constructions de couvents et d'ouvrages de fortifications ${ }^{11}$. La ville de Nîmes et le Midi de la France dans son ensemble regorgent de ruines qui sont déterrées et viennent ensuite alimenter les cabinets à travers tout le royaume. La décennie de 1620 à 1630 marque de ce point de vue une période particulièrement foisonnante pour Nîmes, avec les démolitions des fortifications qui succèdent aux guerres de Rohan et donnent lieu à de multiples trouvailles archéologiques.

5 Toutes ces «trouvailles" sont présentées par Rulman et les circonstances de leur découverte sont bien souvent rapportées tout au long de ses récits ${ }^{12}$. La Tête de Plotine femme de l'empereur Trajan fut ainsi «trouvée aux mouvements derniers, entre les portes de la Bouquerie, et celle des Precheurs, 3 pieds au dessous d'un pavé, fait à la musaïque ${ }^{13}$ ». À cette pièce s'ajoute un Busc de la Lupercale, trouvé pour sa part à « deux toises pres de la Plotine ${ }^{14} »$. La découverte de la Statue de la déesse de la Concorde suscite pour sa part une longue digression rendant compte de l'intérêt qu'a suscité cette pièce dégagée en 1622 : «le baron de Brison ${ }^{15}$ qui commandoit dans Nismes, faisant le tour des fortifications, courut au bruit confus qui sortoit du fossé du bastion du château dès qu'on eut fait la découverte de cette statuë. Il en donna deux pistoles à ceux qui l'avoient trouvée : et la consigna des mains d'Abrenathée principal du collège ${ }^{16}$, pour l'enrichir de ses armes au dessus, et au dessous d'un exact devis de ses perfections ${ }^{17}$ ». Citons encore parmi les statues et autres pièces sculptées, un Hercule de marbre, trouvé «dans le fossé d'un 
bastion ", sans plus de précision cette fois-ci ${ }^{18}$. Outre les statues et figurines, l'auteur répertorie encore toutes sortes d'objets du quotidien, comme cette « urne de verre [...] trouvée avec 4 larmoirs de verre commun, deux petits vases, et deux lampes de terre scellée, qui étoient à l'entour, dans un monument rond ${ }^{19}$ ". À d'autres occasions, ce ne sont plus seulement les travaux des fortifications qui sont évoqués, mais d'autres circonstances, comme les fortes pluies qui permettent aux bagues et autres pierres précieuses de remonter à la surface du sol : « lorsque la fontaine deborde, qu'elle ruisselle partout, et regorge dans le cadarau, elle mene toujours sur le gravier, et laisse à sec quelque chose de rare \& de nouveau qui se trouva après que les eaux se sont retirées ${ }^{20}$ ». Ce qui est vrai pour les pierres, l'est aussi pour les médailles, avec, par exemple, ces cinq mille médailles d'argent déterrées par deux ouvriers nîmois ${ }^{21}$. À la lecture de ces quelques exemples, on mesure l'importance des découvertes archéologiques qui sont tout sauf anodines pour les collectionneurs méridionaux.

6 Toutes ces trouvailles alimentent en effet les cabinets des collectionneurs du Sud de la France, ce dont le texte de Rulman rend parfaitement compte. À l'échelle de Nîmes tout d'abord, plusieurs individus émergent. Le premier d'entre eux, que Rulman distingue à diverses reprises, est le greffier Préneuf. Décédé à l'heure où l'auteur rédige ses Récits et son Inventaire, il est dépeint comme celui qui était de son vivant « le mieux assorti de tous en fait de sépulchres, urnes, \& autre dépendances des funerailles ${ }^{22}$ ». Son cabinet est conservé par ses héritiers après sa mort et notamment ses urnes qu'il possédait en grand nombre : en verre, de différentes couleurs, mais aussi une en albâtre « reluisante comme une glace de $\operatorname{cristal}^{23}$ ", des vases et des lampes dont plusieurs sont représentés dans le recueil de dessin ${ }^{24}$. Il avait encore des médailles ${ }^{25}$, de nombreuses pièces dont une statue de Mercure trouvée « dans le fondement du logis, qu'il a bâti tout contre la vieille porte romaine qui paroit encore en son entier au chemin de Montpellier ${ }^{26} »$, un Adonis en terre cuite, un Cupidon ou un dieu canope de bronze ${ }^{27}$. Autant de pièces qui laissent ici présager d'un rassemblement d'objets antiques assez important.

7 Vient ensuite Tournier, contrôleur du domaine, qui est cité à plusieurs reprises et dont on reconnaît également des pièces au sein du recueil de dessins ${ }^{28}$. Rulman évoque par exemple « une belle urne ronde de verre ${ }^{29}$ et d'autres vases et fioles ${ }^{30}$ abrités dans son cabinet. C'est lui qui s'empare de la fameuse Tête de Plotine ${ }^{31}$ et qui achète le Busc de la Lupercale $^{32}$. Mais Rulman le retient surtout pour ses médailles, rappelant ainsi qu'il en est le « depositaire des plus importantes, et en plus grand nombre » ${ }^{33}$. Ce même Tournier est par ailleurs mentionné en 1649 par Pierre Borel (1621-1671), qui dresse une liste des principaux cabinets de curieux européens, sans plus de détails, alors que dans ces mêmes années, précisément en 1655, un autre antiquaire nîmois, Gaillard Guiran (1600-1680), salue à son tour le goût de Tournier pour les médailles ${ }^{34}$. Ces quelques témoignages signalent ici un véritable collectionneur d'antiquités.

8 Véritable collectionneur d'antiquités car, par l'intermédiaire de personnalités comme Tournier ou le greffier Préneuf, l'on touche à un problème méthodologique majeur face à une source comme celle de Rulman : dans sa ville de naissance, ce notable parfaitement établi connaît tous les acteurs qui, de près ou de loin, gravitent autour des découvertes et se saisissent des vestiges. Son recensement des amateurs nîmois acquiert de ce fait un caractère exhaustif et, pour une part d'entre eux, les noms fournis apparaissent inédits ou tout au moins méconnus. Mais, dans un tel contexte, il est difficile de distinguer les rassemblements qui relèvent de l'opportunité et de la réunion fortuite d'objets, de ceux qui relèvent de la collection qui, elle, « cesse d'être une accumulation sans discernement ${ }^{35}$ 
». Si l'on peut donc dégager des protagonistes en ce début de XVII ${ }^{e}$ siècle, comme Tournier, d'autres sont plus délicats à appréhender, comme peut-être le greffier Préneuf dont bon nombre de pièces dépendent justement de trouvailles fortuites, à l'image de son Adonis en terre cuite, qu'il trouva lui-même dans un fossé, ou de son Mercure en terre cuite trouvé «dans le fondement de son logis ${ }^{36}$ ». Dans cette même perspective, d'autres personnalités sont signalées pour quelques pièces, sans que l'on puisse déterminer l'ampleur de leur rassemblement. Il y a tout d'abord Ducray, apothicaire, propriétaire d'un buste d'enfant, d'une petite urne ou encore de quelques écuelles, d'anses ${ }^{37}$. Jacques Le Blanc, sieur de la Rouvière, juge des conventions et de la cour royale ordinaire, est de son côté mentionné pour quelques pièces, dont un bas-relief représentant, d'après Rulman, une Dame romaine et son affranchi ou encore un Demi corps d'Harpocrate ${ }^{38}$. C'est aussi dans la maison de l'un de ses parents que l'on a retrouvé l'Apollon tenant la tête d'Esculape ${ }^{39}$. Le cabinet du docteur d'Arbouse (ou d'Arboux) présente un bel ensemble d'urnes, vases et fioles ${ }^{40}$, alors que le médecin Pistori, décrit comme un "homme de sçavoir, et curieux », est quant à lui propriétaire d'un Hercule de marbre $e^{41}$; on le retrouve, avec d'autres, cité en tant que propriétaire de plusieurs médailles ${ }^{42}$. Des figures multiples qui profitent sans doute pour une part d'entre elles de la proximité des vestiges et des découvertes archéologiques.

9 Au-delà de Nîmes, le témoignage de Rulman permet de retracer le parcours de nombreuses pièces d'antiquités issues du sol de la ville et qui alimentent non seulement les cabinets d'amateurs locaux, mais également ceux du Languedoc ou du royaume. À l'image de l'ingénieur d'Argencourt ${ }^{43}$ qui, passant à Nîmes en 1622, se saisit d'une urne d'albâtre auprès d'une "femme du commun » et la transmet ensuite au cabinet de Monsieur, frère du roi, Gaston d'Orléans (1608-1660) ${ }^{44}$, ou du marquis de Grimaut qui s'empare de mille médailles trouvée en 1621 et les dépose lui aussi dans le cabinet de Monsieur ${ }^{45}$. À l'échelle du Languedoc, les objets circulent à leur tour, comme cette Tête de Caius Marius abritée au sein du cabinet du médecin et collectionneur montpelliérain François Ranchin (1560-1641) ${ }^{46}$. Cette Tête présentée comme « tant fameuse » par l'auteur, a été trouvée dans les ruines des fortifications où elle a été prélevée par un cordelier nîmois, Brossan, qui la remet au prieur de Saint-Bauzile et passe, à la mort de ce dernier, dans le cabinet du montpelliérain ${ }^{47}$. On retrouve ce même Brossan, propriétaire d'une Teste de Commode, mais qui s'en tient sans doute beaucoup plus au rôle d'intermédiaire et se contente de faire passer ses découvertes aux curieux du pays, comme l'Hercule de marbre, qu'il transmet au médecin Pistori, ou ici cette Tête de Caius Marius ${ }^{48}$. Les découvertes de pièces d'antiquités passent donc de main en main et de collection en collection, avec des acteurs et intermédiaires qui se distinguent et reviennent régulièrement au fil du récit de Rulman.

10 Toutes ces trouvailles rapportées par le Nîmois illustrent parfaitement l'activité foisonnante qui prend place dans les territoires du Midi tout au long de l'époque moderne. Elles renforcent encore l'image d'un milieu d'antiquaires et de curieux d'antiquités particulièrement vivace en ce début de XVII ${ }^{e}$ siècle. Mais elles interrogent en même temps la nature de cette activité qui relève autant de circonstances favorables que de véritables démarches de curieux et collectionneurs. De toute évidence, les attitudes, à l'instar de personnalités comme Tournier ou Préneuf, sont variées et une lecture attentive du texte de Rulman permet de rendre compte de cette complexité, notamment lorsqu'il précise que "ceux qui par expérience sçavent les endroits fortunés, où le courant de l'eau jette ses tresors y vont comme à la moisson d'or, assurés de récompenser 
leurs peines par leur bonheur ${ }^{49} »$ : autour des découvertes, se mêlent donc les uns, qui guettent la pièce rare pour compléter leur rassemblement, et les autres, qui profitent d'une profusion d'objets soit pour réunir quelques pièces éparses, soit pour les faire passer et les vendre. C'est alors que se dessine peu à peu un réseau de curieux qui constituent une élite antiquaire locale et régionale.

\section{Le milieu antiquaire de la France méridionale}

11 La constitution de réseaux au sein de la République des Lettres a fait l'objet d'études récentes ${ }^{50}$. Parmi les collectionneurs et curieux, la correspondance, la diffusion des objets, des dessins et, avec elles, le fait d'entretenir et de cultiver ses relations était devenue caractéristique : la figure de Peiresc, qui polarise une grande part des échanges, semble en ce sens à elle seule symboliser tout le tissu méridional de ce début de XVII ${ }^{\mathrm{e}}$ siècle. Mais les exemples sont nombreux et, à la fin du XVI siècle, on voit notamment Thomas Platter (1574-1628) profiter de sa visite à Montpellier pour se rendre dans le cabinet du médecin Laurent Joubert (1529-1583), alors en partie conservé par son fils, et de Richer de Belleval (v. 1564-1632), où il signale les plantes, les objets d'art et autres vieilles choses qui attirent son attention ${ }^{51}$. Certains collectionneurs n'attendent pas d'être visités et consignés par les voyageurs, pour faire connaître leur collection et en publier un catalogue, comme Bernard Palissy (v. 1510 - v.1590) en $1580^{52}$. Au début du XVII siècle, cette pratique se poursuit et s'intensifie, avec les catalogues des provençaux François Du Périer ( ? - 1623) et Antoine Agard (1585-1609), qui datent respectivement de 1608 et $1611^{53}$.

12 S'inscrivant ainsi directement dans cette pratique, Rulman accorde plusieurs descriptions aux collections d'amateurs languedociens. Il décrit tout d'abord le cabinet narbonnais de Pierre Garrigues (1560-1643), ingénieur du roi, chargé de la restauration des fortifications de Narbonne, dont il est également consul en 1604. Garrigues est aussi connu en tant qu'antiquaire, auteur notamment de deux manuscrits consacrés aux inscriptions et basreliefs de sa ville, avec des représentations de certaines pièces ${ }^{54}$. En tant que collectionneur, il n'est pas resté inconnu puisqu'à la suite de Rulman, Pierre Borel le mentionne dans sa liste des principaux cabinets ${ }^{55}$.

13 La description de Rulman ne laisse en effet aucun doute sur l'importance du cabinet de Garrigues qui n'a rien du simple rassemblement fortuit. Il fait ainsi état d'un « riche cabinet » et de pièces ou revers de médailles "si bien choisis qu'ils meritent d'estre recités ${ }^{56}$ ». Les observations détaillées de Rulman, tout comme ses remarques à propos des objets décrits, laissent entendre qu'il les a bien vus, ou tout au moins, qu'il en a obtenu un rapport circonstancié. Pièces de reliefs, de bronze, gravures, médailles et autres pièces rares sont ainsi toutes présentées, souvent de manière laconique, mais parfois agrémentées d'un court rappel de l'épisode mythologique qu'elles représentent ou d'appréciations plus personnelles. Dans son Recueil d'antiquités de la ville de Narbonne, Garrigues a par ailleurs laissé des dessins de certaines de ses pièces de collection. Y figurent la représentation d'un triclinium sous la forme "d'une grosse pierre commune, representant une table a trois angles esgaux, le vin \& le verre, le pain \& cousteau dessus, avec les deux figures du mari \& de la femme, qui paroissent assez combien qu'elles sont corrompeues pour estre recogneues ${ }^{57}$ ", ou encore celle d'une "corneline en quarré berlong de la longueur du travers de pouce ${ }^{58} »$. La rencontre entre les deux hommes ne se limita pas au seul cabinet de Pierre Garrigues, ce dernier renseignant également le Nîmois 
sur les vestiges à observer dans la ville. À la lecture de ces passages, on mesure parfaitement la dimension collective du travail d'antiquaire.

De ville en ville, on sert de guide ou l'on se fait guider : "voici la suite de ce que nous avons veu de rare étants sur les lieux, apprins de la bouche de Garrigues ou recueilli d'ailleurs ", précise ainsi Rulman ${ }^{59}$. Cette transmission est ici plus orale que livresque, elle favorise les liens et les marques de respect entre ces hommes que les intérêts, la curiosité et le goût rassemblent. Outre la mention de son « riche cabinet », Rulman dira encore à propos de Garrigues et de ses actions en faveur des antiquités narbonnaises : «Car elle [Narbonne] fût honnorée en son tems de la magnificence d'un amphithéâtre qui est entièrement aplani et son sol tombé entre les mains de Garrigues l'ingénieur et très intelligent aux choses antiques qui travaille depuis longtems à le faire remonter du sépulchre qui l'a englouti ${ }^{60} \%$. Avec les objets, ce sont tous les savoirs des antiquaires sur leur ville, sur la localisation et l'histoire de chaque pièce digne d'intérêt, qui se diffusent et circulent parmi cette élite.

Le même procédé peut s'observer à l'égard du chancelier de l'université de médecine de Montpellier, François Ranchin (1564-1641). Collectionneur reconnu en son temps, Ranchin est signalé dans diverses sources : par Pierre Borel au milieu du XVII siècle, par Jacob Spon et Charles César Baudelot de Dairval à la fin du siècle ${ }^{61}$. Il possède en effet à Montpellier une importante collection d'antiquités dont une partie, notamment des pierres gravées, provient d'Henri d'Angoulême (1551-1586), fils naturel du roi de France Henri II, gouverneur de Provence à la fin de sa vie ${ }^{62}$. Elle comptait par ailleurs pas loin de quinze mille médailles - soit l'un des rassemblements les plus importants qu'ait observé Antoine Schnapper ${ }^{63}$ - et suscita l'intérêt de Peiresc : les deux hommes correspondent à diverses reprises, en $1614^{64}$ et surtout à partir de 1628, lorsque Ranchin souhaite vendre son cabinet ${ }^{65}$. Chez Rulman, les pièces conservées par Ranchin font l'objet d'une longue description mais également de quelques représentations ${ }^{66}$, comme la Tête de Caius Marius évoquée plus haut, ou l'Homère entre ses deux filles offert à Ranchin par le "grand connetable de France son maitre et son bienfaicteur", Henri $I^{\text {er }}$ de Montmorency (1534-1614), que Ranchin avait soigné en $1598^{67}$. Cette collection rassemblée par Ranchin lui vaut également des commentaires élogieux: il est dépeint comme un homme de « cognoissance profonde de tout ce qui depend de l'antiquite romaine» alors que son cabinet contient «des plus rares statues, de plus belles testes de meilleures figures, de plus grand nombre de medailles, et de plus excellentes graveures, qu'on puisse voir dans toute les villes de la province; quoy qu'il y en aye, en plusieurs autres villes qui en sont bien assorties ${ }^{68}$ ». Le don qu'il fait de sa Chaire de marbre au profit de la faculté de médecine suscite encore les louanges de Rulman : « Parce que son cabinet est remply d'un monde de merveilles, et que c'est un ciel enrichi de l'éclat des planettes, et de brillantes confusions d'une infinité d'estoilles. Il a voulu logé ceste belle chese de marbre antique dans son auditoire du College de medecine, \& au millieu des degres de la chapelle de l'anatomye ${ }^{69} »$. Plus tard, dans son Plan des cuvres mêlées, Rulman le qualifie encore de " parangon des bons antiquaires ${ }^{70}$ ».

16 Parmi les personnalités languedociennes, d'autres noms sont à leur tour cités. À Montpellier toujours, est mentionné Laurent Catelan (v. 1567-1647) apothicaire et collectionneur connu pour ses « raretés modernes qui distinguent son esprit curieux, \& son humeur sortable aux étrangers d'avec le commun des autres apoticaires ${ }^{71}$ ». Un Mars en bronze lui appartenant est également inséré dans le volume de dessins ${ }^{72}$. On voit encore à la fin de ce même volume, des représentations de pièces du cabinet de Philippe 
(1571-1627) et Simon de Bornier (1604-1648), père et fils, qui n'ont en revanche pas donné lieu à des descriptions circonstanciées dans le corps du texte, à l'exception de quelques mentions comme une sculpture signalée dans leur domaine de Teillan, sans guère de détails : «Ceste ci est a Teillian, belle maison champetre du feu president Bournier, bâtie à la moderne et assise entre Nismes et Montpellier. Il l'enrichissoit tous les jours des plus belles pieces antiques qu'il pouvoit recouvrer dans la province ${ }^{73}$ ». Les commentaires qu'ajoute Rulman à leur intention dans son Plan des ceuvres mêlées, montrent qu'il connaissait néanmoins bien cette famille. Les neufs grosses colonnes qui enrichissent leur jardin font ainsi voir au monde, "que l'esprit de cet homme [Philippe Bornier] n'estoit pas de la trempe du commun, que ses visions etoient sublimes, sa connoissance universelle, son humeur obligeante, sa mort prejudiciable au public et sa memoire de fort bonne ardeur parmi les gens de condition ${ }^{74}$ ». Là encore, la reconnaissance et l'éloge sont de mise.

17 À travers ces différentes descriptions, deux points méritent donc d'être soulignés. D'une part, autour de ces personnages d'envergure comme Ranchin ou Garrigues se révèle une élite antiquaire régionale qui met en lumière des collectionneurs languedociens peut-être plus en retrait - en terme d'antiquités - que le milieu provençal gravitant autour de la figure de Peiresc, mais néanmoins tout à fait actifs et en relation avec des personnages de haut rang. D'autre part, à travers la constitution de ces réseaux actifs transparait la fonction «sociale » d'un cabinet: lorsque le rassemblement est apprécié pour ses objets «si bien choisis» ou «rares", la collection mérite d'être signalée et connue, sa réputation rejaillissant alors sur son propriétaire. Ainsi, tout homme qui témoigne par ses actions d'une révérence à l'égard des vestiges antiques et participe à une meilleure connaissance de l'Antiquité, suscite l'estime de ses pairs.

Autour de ce réseau d'antiquaires et curieux d'antiquités qui se connaissent et se reconnaissent entre eux, s'élabore alors une élite qui se revendique peu à peu comme telle au sein de la République des Lettres. Chez Rulman, elle se révèle particulièrement dans ses discours d'introduction, et notamment dans celui qui ouvre son troisième livre essentiellement consacré aux descriptions d'antiquités des cabinets du Sud de la France ${ }^{75}$. Y est clairement distingué «le commun des hommes » - qui selon l'auteur, trouve du plaisir « au recit et a l'oüye des choses inutiles, a la conversation des personnes oiseuses, et au rendésvous des lieux publics, où le tems se perd, et s'employe le plus souvent a mal penser, et mal parler d'autrui » - et les " curieux », " esprit libres et clairvoyants » qui eux, « divisent leurs pensées [...] par l'ordre d'un jugement solide prescrit à leurs paroles, \& à leurs actions ». Sous-jacente, se lit également une légitimation des occupations des curieux, dressant un portrait d'hommes de raison, qui tout en s'emparant de pensées plus hautes que celles du " commun », n'en oublient pas les tâches afférentes à leur charge ou profession. À eux seuls est réservé le droit d'observer les antiquités les plus dignes, comme l'exprime parfaitement Rulman à l'égard des pièces transportées dans les cabinets, « reservées à l'entreveüe des plus curieux, [...] : ce sont les otages \& les images parlantes de leur antiquité romaine [...] et de la delicatesse curieuse des esprits judicieux qui les ont conservées en l'état que le temps et les calamitez publiques les ont mises ". C'est encore l'expression et la conceptualisation d'un patrimoine commun qui ressort lorsqu'il lance: «Je ne veux rien obmettre si je puis de ce qui est en nature parmi nous, où qui l'a été du temps de nos peres, qui ont eu quelques avant goût des merveilles que nous avons decouvertes dans les abîmes de nos fortifications, et les desolations humaines ", déplorant dans le même temps, la détérioration de certains vestiges nîmois, 
du fait du «mépris que font la plus part des Hommes de ce temps des choses si belles; ne leur êtant pas presentement utiles et de pieces si rares, qui ne leur sont pas comodes ${ }^{76}$ ». Une élite antiquaire qui se retrouve donc par l'intermédiaire d'une curiosité et se reconnaît aussi bien à travers des pratiques et des intérêts partagés qu'à travers un passé commun qui va puiser ses origines dans des temps lointains où les Romains font figure d'ancêtres prestigieux.

\section{De la Gaule Narbonnaise aux provinces de l'Ancien Régime : la persistance d'une identité locale}

Les antiquaires, à leur manière, se sont emparés de l'histoire de leur région. Mais comme le note Arnaldo Momigliano, leurs ouvrages «n'aboutissaient jamais, en tout état de cause, à un livre d'histoire ordinaire ${ }^{77}$ ». Et plus encore, l'importance qu'ils ont accordée aux témoignages non littéraires d'une part, et la place majeure qu'occupait la quête des origines d'autre part, révèlent « le besoin de nouvelles histoires » qui a émergé avec l'ère des antiquaires ${ }^{78}$. Dans les années quatre-vingt-dix, Pierre Pinon montrait comment les érudits du $\mathrm{XVI}^{\mathrm{e}}$ siècle, tout en s'intéressant aux origines du pouvoir, tentaient de «magnifier la France et son identité » en ne se rattachant plus seulement aux Francs ou aux Romains, mais en allant puiser plus loin, chez les Gaulois notamment, la naissance de leur nation ${ }^{79}$. Il rappelle alors que, «dans la mentalité de l'époque, une nation, une ville, un groupe social quelconque tire non seulement son importance de sa puissance présente, mais aussi de son ancienneté ${ }^{80}$ ». De son côté, Krzysztof Pomian distingue deux pôles, l'un classique et l'autre ethnique ou local. Il constate entre eux une différence de statut : le premier fait l'objet d'une admiration, alors que le second sert bien souvent un intérêt patriotique ${ }^{81}$. À travers de nombreux ouvrages portant sur l'Antiquité, l'Histoire ou les Mémoires selon les appellations, de telle ville ou province, s'opère ainsi une véritable redéfinition du passé à laquelle l'œuvre de Rulman adhère pleinement. Sans que s'instaure une opposition entre ces deux pôles, l'auteur nîmois juxtapose en effet à plusieurs reprises les références aux Romains (pôle classique) et aux Gaulois (pôle local) ${ }^{82}$. Les raisons politiques et idéologiques qui sous-tendent cette redéfinition du passé sont multiples. Frédérique Lemerle y décèle un moyen de s'affranchir de la prédominance de l'Italie et de son glorieux passé, mais aussi la manifestation d'une époque au cours de laquelle le roi de France tente d'asseoir son autoritée ${ }^{33}$. En même temps, la haute Antiquité des civilisations préromaines entretient le patriotisme local, en prouvant l'ancienneté et donc le prestige d'une ville, et par là même, en justifiant ses privilèges ou son indépendance ${ }^{84}$.

20 Le projet d'écriture de Rulman est en ce sens tout à fait intéressant : à une période où les histoires locales prédominent, le Nîmois en appelle à nouveau à l'héritage antique en circonscrivant les limites de son étude non pas aux confins d'une ville ou d'une province, mais aux anciens territoires de la Gaule Narbonnaise. La Gaule Narbonnaise de Rulman lui permet ainsi de s'emparer d'un territoire allant de Toulouse à Nice et en remontant jusqu'à Vaison-la-Romaine. Parmi les grands sites étudiés, l'on retrouve Toulouse, Narbonne, Arles, Orange, Marseille, Fréjus et, bien sûr, Nîmes, mais aussi des sites moins fréquemment relevés comme Béziers. Il ne va en revanche pas au-delà de Narbonne et ignore toute la partie au Nord de Vaison-la-Romaine, notamment Vienne. Dès les premiers folios de son discours, il confère à la Narbonnaise un statut de référent identitaire dans ce Midi de la France du XVII ${ }^{e}$ siècle. Il se distingue au passage de ses 
pairs : «Je fais pour la Gaule Narbonnoise ce que peu de gens ont osé entreprendre pour l'Italie. \{Et pour Nismes ce que quelques-uns ont voulu essayer pour Rome.\} Je le fais dans le sentiment de mon affection, comme citoyen dans l'honneur, et l'intérest de ceste corone comme François, $[. . .]^{85}$ ». Néanmoins, au-delà de ces notes d'intention, l'auteur replace presque systématiquement sa ville d'origine et sa province du Languedoc au premier plan. Si la Narbonnaise a par exemple " esté estimée une seconde Italie », Nîmes passe quant à elle pour « l'abbregé de Rome ${ }^{86}$ ». Dans cette perspective, la place accordée aux villes et antiquités de Provence apparait beaucoup plus réduite que celle que le titre de son ouvrage - Récit des anciens monumentz qui parroissent encore dans les despartementz de la première, \& seconde Gaule Narbonoise... - pouvait annoncer. Rulman leur consacre en effet une trentaine de récits, tous placés dans son premier livre, laissant le reste de ce livre soit environ une soixantaine de récits - au Languedoc, alors que Nîmes mobilise à elle seule les deux livres suivants ${ }^{87}$.

Bien que ces quelques trente récits ne soient pas négligeables, l'attitude du Nîmois ne diffère guère de celle de ses contemporains, avec une référence à la Gaule Narbonnaise et à son espace qui sert plus de symbole que de véritable fondement identitaire. Déjà chez Jean Poldo d'Albenas (1512-1563), la Narbonnaise n'était que citée dans le cœur de son étude, puis reléguée à la fin de son ouvrage dans une Table des noms, par ordre de l'Alphabeth, des lieux, villes, fleuves \& montaignes, les plus mémorables de la Province Narbonoise...8 ${ }^{88}$. Plus tard, en 1633, le Toulousain Guillaume Catel (1560-1626) adopte une organisation similaire à celle de Rulman et n'octroie à l'ancienne province romaine que quelques chapitres d'introduction avant de se concentrer sur le Languedoc ${ }^{89}$. On peut dire la même chose d'antiquaires provençaux comme Jules-Raymond de Solier (v.1530-1595), qui s'attarde davantage sur la filiation entre Marseille et les Phocéens, Antoine Ruffi (1607-1689) et un peu plus tard Honoré Bouche (1598-1671), qui se contentent d'évoquer leur province dans les premiers chapitres de leurs ouvrages respectifs ${ }^{90}$. La Gaule Narbonnaise sert de dénominateur commun chez tous ces amateurs d'antiquités du Midi, mais elle ne prend le pas ni sur le pôle provincial et ses origines, légendaires ou non, ni sur le pôle local. À l'instar des Marseillais qui invoquent fréquemment leur filiation avec les Phocéens, les Nîmois, quant à eux, se réclament d'un fils d'Hercule - Nemausus - que le héros mythologique aurait eu à son retour de la péninsule ibérique et qui expliquerait l'origine du nom de la ville ${ }^{91}$. Cette ascendance leur permet ainsi de remonter aux temps mythologiques et de dépasser l'héritage romain en préférant les Gaulois; le surnom de "grand Hercule gaulois ${ }^{92}$ utilisé par Rulman pour désigner Hercule est en ce sens tout à fait signifiant. À chaque fois, le processus identitaire local et régional - ici Nîmes et la province du Languedoc - l'emporte sur une identité méridionale qui se déploierait dans les contours et l'héritage de l'ancienne province narbonnaise.

L'étude des manuscrits de Rulman vient donc confirmer l'image d'un milieu d'antiquaires et d'amateurs d'antiquités particulièrement actif en ces premières décennies du XVII ${ }^{e}$ siècle. Elle permet en même temps de mettre en lumière des protagonistes nîmois et languedociens parfois restés plus en retrait - pour cette période du moins - face à leurs homologues provençaux dont les collections, qu'il s'agisse des Aixois Peiresc ou Borrilly (1564-1648) ou de l'Arlésien Agard, ont désormais été largement étudiées. La commodité des trouvailles qui favorise sans doute une partie des vocations n'empêche pas l'apparition de figures majeures. Les liens étroits qui unissent une personnalité comme Rulman avec d'autres, comme Garrigues à Narbonne, Ranchin à Montpellier et, dans une certaine mesure, avec le Nîmois Tournier, montre que se constitue là une élite de curieux 
d'antiquités qui se reconnaissent entre eux et entretiennent des réseaux où les pièces circulent - à l'image de ce poinçon offert en présent par Garrigues à Ranchin - où l'échange des connaissances se produit dès que l'occasion se présente - comme Garrigues encore, qui fait part à Rulman des antiquités à observer dans sa ville de Narbonne. En outre, les textes de Rulman laissent apercevoir l'élaboration d'une identité méridionale partagée par ces curieux qui se reconnaissent dans un passé commun inscrit dans les territoires de la Gaule Narbonnaise. Cette identité méridionale s'efface cependant face à des enjeux plus locaux, enracinés dans la ville de naissance de chacun. La chose est patente chez Rulman, qui embrasse des territoires comprenant l'ensemble des régions méditerranéennes, mais qui reste irrémédiablement fixé sur sa ville, Nîmes, et sa région, le Languedoc, qui occupent tous deux l'essentiel de son étude. C'est également cet enracinement dans le Bas-Languedoc qui explique le peu de relations qu'il entretient avec les curieux de Provence, ce qui souligne encore la coexistence dans le Midi français de deux identités, avec, d'un côté, des écrits consacrés au Languedoc et, de l'autre, à la Provence. En somme, si le passé romain agit comme un référent incontournable dans ces textes d'antiquaires, il demeure encore largement empreint des enracinements locaux de leurs auteurs.

\section{NOTES}

1. Arnaldo Momigliano, Problèmes d'historiographie ancienne et moderne, Paris, Gallimard, 1983, p. 252.

2. Ibid., p. 252.

3. Ibid., p. 283.

4. Antoine SCHNAPPER, Le Géant, la licorne et la tulipe. Collections françaises au XVII ${ }^{e}$ siècle. Histoire et histoire naturelle. I, Paris, Flammarion, 1988, p. 180.

5. Ibid., p. 249.

6. Véronique KRINGS et Catherine VALENTI, dir., Les Antiquaires du Midi. Savoirs et Mémoires XVI ${ }^{e}$-XIX siècle, Paris, Errance, 2011, p. 7.

7. Voir Pierre GRos, «Un programme augustéen, le centre monumental de la colonie d'Arles », Jahrbuch des Deutschen Archäologische Instituts, vol. 102, 1987, p. 339-363. Sur Arles et Nîmes sous Auguste, voir encore l'article du même auteur, Pierre Gros, « Les villes d'Auguste en Narbonnaise. Nouvelles recherches sur Arles et Nîmes ", in Michel Christol et Dominique Darde, dir., L'Expression du pouvoir au début de l'Empire. Autour de la Maison Carrée à Nîmes, Actes de colloque (Nîmes, Carré d'Art, 20-22 octobre 2005), Paris, Errance, 2009, p. 111-117.

8. Sur Orange et Vienne, voir Frédérique LEMERLE, La Renaissance et les antiquités de la Gaule. L'architecture gallo-romaine vue par les architectes, antiquaires et voyageurs des guerres d'Italie à la Fronde, Brepols, Turnhout, 2005, p. 93-97.

9. Bibliothèque nationale de France (désormais BnF), fonds français, ms 8648-8651 et Bibliothèque du Carré d'Art de Nîmes (désormais BCAN), manuscrits occidentaux, ms 179-183.

10. BnF, ms 8648. Citons aussi une deuxième partie contenant des dessins d'antiquités de la ville de Béziers et de ses alentours. 
11. Voir Pierre PINON, «Les pratiques de l'archéologie et les circonstances des découvertes au XVI e et au début du XIX ${ }^{\mathrm{e}}$ siècle ", in Jean-Pierre Demoule et Christian Landes, dir., La Fabrique de l'archéologie en France, Paris, La Découverte, 2009, p. 34-53.

12. BnF, ms 8651, voir respectivement les récits aux f. 168, 174, 184, 195, 229 et 264. Certains de ces récits ne contiennent cependant pas de textes, et ne présentent que des titres. Voir aussi BCAN, ms 179, f. $93 \mathrm{r}^{\circ}$ et suivants.

13. BnF, ms 8651, f. $101 \mathrm{v}^{\circ}$. Il s'agit ici du secteur de l'actuel boulevard Gambetta, Rulman date précisément cette découverte de 1625.

14. BnF, ms 8651, f. $102 \mathrm{r}^{\circ}$. Voir au sujet de ces deux pièces, Jean-Luc Fiches et Alain VeYrAC, Carte archéologique de la Gaule. Nîmes. 30/1, Paris, Académie des Inscriptions et Belles-Lettres - Ministère de la Culture, 1996, p. 386.

15. Joachim de Beaumont (? - 1628), baron de Brison, gouverneur de Nîmes. À son sujet, voir notamment François-Alexandre AUBERT DE LA CHESNAYE DES BOIS, Dictionnaire de la noblesse (...), Paris, La Veuve Duchesne, 1771, t. II, p. 243.

16. Adam Abernethy, presbytérien issu d'une famille noble écossaise, se fit naturaliser français et appeler Abrenethée. Diplômé de l'université d'Edimbourg en 1594, il rejoint le Languedoc et Nîmes où il enseigne au début du siècle. Voir à son sujet, Pierre AzAïs (l'Abbé), «Le Collège de Nîmes ", Mémoire de l'Académie de Nîmes, VII ${ }^{\mathrm{e}}$ série, t. 1, Nîmes, Clavel-Ballivet, 1879, p. 133-255 et plus précisément p. 204-206.

17. BnF, ms 8651 , f. $113 \mathrm{v}^{\circ}-114 \mathrm{r}^{\circ}$.

18. Ibid., f. $121 \mathrm{v}^{\circ}$.

19. BnF, ms 8651, f. $218 \mathrm{v}^{\circ}$. Voir aussi Jean-Luc Fiches et Alain VeYrac, Carte archéologique... op. cit., p. 520.

20. $\mathrm{BnF}, \mathrm{ms} 8651$, f. $229 \mathrm{r}^{\circ}-\mathrm{v}^{\circ}$. Comprendre en réalité «Cadereau » qui désigne un ruisseau à sec qui se remplit lors de pluies intenses. À Nîmes, il se situe aux abords et au sud des Jardins de la Fontaine.

21. Ibid., f. $237 \mathrm{v}^{\circ}$.

22. BCAN, ms 180 , t. 2 , p. 184.

23. Ibid., p. 256.

24. BnF, ms 8648, f. $79 \mathrm{r}^{\circ}$ et $80 \mathrm{r}^{\circ}$.

25. BCAN, ms 180 , t. 2, p. 300.

26. Ibid., p. 184-185. Il s'agit de la Porte de France.

27. Ibid., p. 187-189. On trouve encore d'autres pièces qui appartiennent au greffier, dans l' Inventaire, $\mathrm{BCAN}, \mathrm{ms} 179, \mathrm{f} .109 \mathrm{r}^{\circ}-\mathrm{v}^{\circ}$.

28. $\mathrm{BnF}, \mathrm{ms} 8648, \mathrm{f} .78 \mathrm{r}^{\circ}$ et $79 \mathrm{r}^{\circ}$. On y reconnaît en effet les patères de Tournier, décrites au $61^{\mathrm{e}}$ récit du ms 8651 .

29. BCAN, ms 180 , t. 2, p. 257.

30. BCAN, ms 179, f. $115 \mathrm{r}^{\circ}-116 \mathrm{r}^{\circ}$, voir encore au f. $107 \mathrm{v}^{\circ}$ et $108 \mathrm{r}^{\circ}$.

31. BnF, ms 8651, f. $101 \mathrm{v}^{\circ}$.

32. Ibid., f. $102 \mathrm{r}^{\circ}$.

33. BCAN, ms 180 , t. 2, p. 300.

34. Pierre BOREL, Les Antiquitez raretez, plantes, minéraux \& autres choses considerables de la ville, \& comté de Castres d'Albigeois \& des lieux qui sont à ses environs, avec l'histoire de ses comtes, evesques, \&c (...), Castres, Colomiez, 1649, p. 129 et Gaillard GUIRAN, Explicatio duorum vetustorum numismatum nemausensium ex aere, Orange, Rabanus, 1655, p. 5.

35. Association Générale des Conservateurs des Collections Publiques (Section Provence-AlpesCôte d'Azur), La Collection: origine, processus, limites à partir de l'exemple de Nicolas-Claude Fabri de Peiresc, Lyon, Fage, 2009, p. 36. Sur ce sujet, l'on renverra à l'ensemble de cet ouvrage, ou encore aux travaux de Krzysztof Pomian. 
36. BCAN, ms 180 , t. 2 , p. 184 et 187.

37. Ibid., p. 197 et p. 254.

38. Ibid., p. 197 et 198. Cette Dame romaine est signalée au logis du juge, mais elle appartiendrait à l'évêque d'après Rulman.

39. Ibid., p. 459.

40. Ibid., p. 259-260.

41. Ibid., p. 189.

42. Ibid., p. 300.

43. Monsieur d'Argencourt, ingénieur du roi, directeur des fortifications de Montpellier. À son sujet, voir notamment David BUISSERET, Ingénieurs et fortifications avant Vauban : l'organisation d'un service royal aux $\mathrm{XVI}^{e}-\mathrm{XVII}{ }^{e}$ siècles, Paris, Comité des travaux historiques et scientifiques, 2002, p. 113 et suivantes.

44. Ibid., p. 253. Sur Gaston d'Orléans, voir Antoine SChNAPPER, Le Géant... op. cit., p. 187 et suivantes.

45. BCAN, ms 180, t. 2, p. 750.

46. Au sujet de François Ranchin, voir Marianne FREYSSINET, François Ranchin et Pierre Gariel : deux Montpelliérains du XVII siècle dans la quête de l'Antiquité, Mémoire de master en histoire de l'art moderne, Montpellier, Université Paul-Valéry, 2008, 131 p. et Flore CÉSAR, Collectionnisme et curiosité à Montpellier, de la Renaissance à l'aube de la Révolution, thèse de doctorat en histoire de l'art moderne, Montpellier, Université Paul-Valéry, 2013, p. 581-603. Voir également Antoine S CHNAPPER, Le Géant... op. cit., p. 247 notamment et Alain CHEVALIER, « Le goût des collectionneurs montpelliérains au XVII ${ }^{\mathrm{e}}$ siècle ", Études sur l'Hérault, n ${ }^{\circ}$, 1993, p. 33-38.

47. BnF, ms 8651, f. $138 \mathrm{r}^{\circ}$.

48. BCAN, ms 180, t. 2, p. 175 et 190.

49. Ibid., p. 267.

50. Voir notamment Véronique KRINGS et Corinne BONNET, S'écrire et écrire sur l'Antiquité : l'apport des correspondances à l'histoire des travaux scientifiques, Grenoble, Million, 2008, $411 \mathrm{p}$.

51. Emmanuel Le Roy LADURIE et Francine-Dominique LieChTENHAN, Le Siècle des Platter. Le voyage de Thomas Platter II, 1595-1599, Paris, Fayard, 2000, p. 220-224.

52. Bernard PALISsY, Discours admirables, de la nature des eaux et fontaines (...), Paris, Martin le Jeune, $1580,361 \mathrm{p}$.

53. François DU PÉRIER, Roolle des medalles et autres antiquitez du cabinet de Monsieur Du Perier, gentilhomme de la ville d'Aix en Provence, s.l., s.d., [c. 1608] et Antoine AGARD, Discours et roole des medailles et autres antiquitez (...) qui ont été recueillies et à présent rangé dans le cabinet du Sieur Agard maistre orfevre et antiquaire de la ville d'Arles en Provence, Paris, [s. n.], 1611 ; rééd. et commenté par Delphine Trébosc, Rennes, Presses universitaires de Rennes, 2007, 148 p.

54. Médiathèque du Grand Narbonne (désormais MGN), Fonds précieux ancien, ms 23, Pierre G ARRIGUES, Inscriptions antiques tumules et epitaphes qui se retrovent en divers endroitz de la ville de Narbonne retirées par Pierre Garrigues de ladite ville, première décennie du XVII ${ }^{\mathrm{e}}$ siècle et $\mathrm{ms} 25$, Pierre GARRIGUES, Recueil d'antiquités de la ville de Narbonne, première décennie du XVII ${ }^{\mathrm{e}}$ siècle.

55. Pierre BOREL, Les Antiquitez raretez,... op. cit., p. 129. Borel précise en effet que le cabinet de Garrigues a été racheté par «Mr. le Baron de Fabresat». À son sujet, voir aussi l'article de Chantal ALIBERT, «Les antiquaires narbonnais à l'origine de la politique patrimoniale de Narbonne ", in Véronique Krings et Catherine Valenti, dir., Les Antiquaires du Midi. Savoirs et mémoires $X V I^{e}-X I X^{e}$ siècle, Paris, Errance, 2011, p. 87-96.

56. BnF, fonds français, ms 8649, f. $109 \mathrm{r}^{\circ}$.

57. Ibid., f. $111 \mathrm{v}^{\circ}$ et dans le manuscrit de Pierre Garrigues, op. cit., MGN, ms 25, f. $82 \mathrm{r}^{\circ}$. On en retrouve une autre copie dans un manuscrit du XVIII ${ }^{\mathrm{e}}$ siècle de l'abbé Bousquet qui s'est penché 
sur l'histoire de Narbonne, MGN, Fonds précieux ancien, ms 24, BousQuET (dit Abbé), Antiquités romaines de la ville de Narbonne, vol. 3 , fo 268 .

58. $\mathrm{BnF}, \mathrm{ms} 8649$, f. $110 \mathrm{v}^{\circ}$ et $\mathrm{MGN}, \mathrm{ms} 25, \mathrm{f} .97 \mathrm{r}^{\circ}$.

59. $\mathrm{BnF}, \mathrm{ms} 8649, \mathrm{f} .46 \mathrm{r}^{\circ}$.

60. BnF, ms 8649, f. $44 \mathrm{v}^{\circ}$. La présence de cet amphithéâtre est effectivement attestée, voir Éric D ELLONG, Carte Archéologique de la Gaule, Narbonne et le Narbonnais 11/1, Paris, Académie des Inscriptions et Belles-Lettres - Ministère de la Culture, 2002, p. 131-132 et Frédérique LEMERLE, La Renaissance et les antiquités de la Gaule... op.cit., p. 62-63 et 84 .

61. Pierre BOREL, Les Antiquitez raretez... op. cit., p. 128 ; Jacob SPON, Recherche des antiquités et curiosités de la ville de Lyon, ancienne colonie des Romains et capitale de la Gaule celtique. Avec un mémoire des principaux antiquaires et curieux de l'Europe, Lyon, Faeton, 1673, p. 222 et Charles de B AUDELOT de DAIRVAL, De l'utilité des voyages et de l'avantage que la recherche des Antiquitez procure aux Sçavans (...), Paris, Aubouin et Emery, 1686, tome II, p. 690.

62. Bibliothèque Méjanes d'Aix-en-Provence (désormais BMAP), ms 211, Lettres de François Ranchin à Nicolas-Claude Fabri de Peiresc, f. 1, Ranchin a en effet acquis des pierres gravées auprès du « grand prieur d'Angoulême, mort gouverneur de Provence en 1586 ».

63. Antoine SCHNAPPER, Le Géant... op. cit., p. 157 et 247, voir également Alain CHEVALIER, Collectionneurs et collections à Montpellier dans la première moitié du XVIII ${ }^{e}$ siècle, Mémoire de D. E. A en histoire de l'art moderne, Paris IV - La Sorbonne, 1987, p. 7 et 19 et Études sur l'Hérault... op. cit., 1993, p. 33-35 ; Marianne FreYsSinet, François Ranchin et Pierre Gariel... op. cit., p. 15-34 et Flore C ÉSAR, Collectionnisme et curiosité à Montpellier,... op. cit., p. 581-603.

64. BnF, fonds français, ms 9538, f. 241-242.

65. Bibliothèque Inguimbertine de Carpentras, ms 1875, f. $425 \mathrm{r}^{\circ}-438 \mathrm{v}^{\circ}$ et BMAP, ms 211, f. 3 et 4. 66. BnF, ms 8648, f. $181 \mathrm{v}^{\circ}-192 \mathrm{r}^{\circ}$ et ms 8651, f. $137 \mathrm{r}^{\circ}$ - f. $154 \mathrm{v}^{\circ}$.

67. BnF, ms 8651 , f. $145 \mathrm{v}^{\circ}$. Ce bas-relief est aujourd'hui visible dans l'actuelle faculté de médecine de Montpellier, dans l'escalier menant à la bibliothèque. Rulman évoque ici simplement le "grand connetable de France ", il pourrait s'agir de François de Bonne, duc de Lesdiguières (1543-1626), protestant converti au catholicisme dans les années 1620, qui était au siège de Montpellier en 1622, et y retourna à plusieurs reprises. Voir à son sujet Louis VIDEL, Histoire de la vie du Connestable de Lesdiguières, Paris, Imprimerie du Roi, 1638. Mais il est plus probable que Rulman évoque là Henri $\mathrm{I}^{\mathrm{er}}$ de Montmorency (1534-1614), connétable de France qui vécut longuement en Languedoc et que Ranchin soigna, comme le rapporte Louis DuLIEU, "Le chancelier François Ranchin », Revue d'histoire des sciences, t. XXVII, n³, Juillet 1974-3, p. 223-239.

68. BnF, ms 8651 f. $137 \mathrm{r}^{\circ}-\mathrm{v}^{\circ}$.

69. Ibid., f. $145 \mathrm{r}^{\circ}$. Cette chaire est aujourd'hui visible dans l'actuel amphithéâtre d'anatomie de la faculté de médecine de la ville.

70. BCAN, ms 180, t. 2, p. 822.

71. BnF, ms 8649, f. $126 \mathrm{v}^{\circ}$. Au sujet de Laurent Catelan, voir Alain CHEVALIER, Collectionneurs et collections à Montpellier,... op.cit., p. 9 et Études sur l'Hérault... op.cit., p. 33 et 36, Alain SCHNAPPER, Le Géant... op. cit., p. 221-222 (entre autres) et Flore CÉSAR, Collectionnisme et curiosité à Montpellier... op. cit., p. 403-414.

72. BnF, ms 8648, f. $185 \mathrm{r}^{\circ}$ avec au bas, la légende « Mars en bronze de Catelan ».

73. $\mathrm{BnF}, \mathrm{ms} 8651, \mathrm{f} .64 \mathrm{r}^{\circ}-\mathrm{v}^{\circ}$. Simon de Bornier possède une collection commencée et transmise par son père Philippe de Bornier (1571-1627), et qui reste dans cette famille durant plusieurs générations. À leur sujet, voir Alain CHEVALIER, Collectionneurs et collections à Montpellier... op.cit., p. 9 et Études sur l'Hérault... op.cit., p. 8-9 et Flore CÉSAR, Collectionnisme et curiosité à Montpellier... op. cit. ,p. 373-383.

74. BCAN, ms 180, t. 2, p. 822 .

75. Ibid., p. 441-443. 
76. Tous les passages cités ici sont tirés de l'introduction du troisième livre des Récits de Rulman. Voir BnF, ms 8651, f. $56 \mathrm{v}^{\circ}$ et suivants.

77. Arnaldo Momigliano, Les Fondations du savoir historique, Paris, Les Belles Lettres, 1992, p. 66.

78. Arnaldo Momigliano, Problèmes d'historiographie,... op.cit., p. 258.

79. Pierre PINON, La Gaule retrouvée, Paris, Gallimard, 1991, p. 14.

80. Ibid., p. 14.

81. Krzysztof Pomian, «Les deux pôles de la curiosité antiquaire», in Annie-France Laurens et Krzysztof Pomian, dir., L'Anticomanie. La collection d'antiquités aux XVIII et XIX ${ }^{e}$ siècles, Actes de colloque (Montpellier-Lattes, 9-12 juin 1988), Paris, École des Hautes Études en Sciences Sociales, 1992, p. 59-68.

82. Voir notamment les dédicaces et le manifeste de l'auteur qui ouvrent ses Récits, BnF, ms 8649, f. $5 \mathrm{r}^{\circ}$ et suivants ainsi que le récit consacré à la fondation de Nîmes, BnF ms 8650, f. $28 \mathrm{r}^{\circ}$ et suivants.

83. Frédérique LEMERLE, La Renaissance et les antiquités de la Gaule... op.cit., p. 53.

84. Ibid., p. 56 et Arnaldo Momigliano, Problèmes d'historiographie... op.cit., p. 274.

85. BnF, ms 8649, f. $23 \mathrm{v}^{\circ}$. Le passage entre les accolades signale une partie de texte rajouté par l'auteur et placé en marge.

86. Ibid., f. $13 \mathrm{v}^{\circ}$.

87. Par «livre", nous reprenons ici l'appellation de l'auteur. Ces trois livres désignent aujourd'hui les trois manuscrits conservés à la $\mathrm{BnF}$, le premier sous la cote $\mathrm{ms} 8649$, le deuxième sous la cote ms 8650 , le troisième sous la cote ms 8651 . Le manuscrit 8648 de cet ensemble comprend pour sa part les dessins d'antiquités qui accompagnent et illustrent les écrits de Rulman.

88. Jean Poldo d'A lBenas, Discours historial de l'antique et illustre cité de Nismes, en la Gaule Narbonoise, Avec les portraitz des plus antiques \& insignes bastimens dudit lieu, reduitz à leur vraye mesure \& proportion, ensemble de l'antique \& moderne ville, Lyon, Roville, 1560 [ 1 ère édition chez le même en 1559], p. 192 et suivantes.

89. Guillaume CATEL, Mémoires de l'histoire du Languedoc curieusement et fidèlement recueillis de divers auteurs (...), Toulouse, Bosc, 1633. Voir précisément les premiers chapitres de son Livre I.

90. Jules-Raymond de SOLIER, Les Antiquitez de la ville de Marseille : où il est traicté de l'ancienne republique des Marseillois : et des choses plus remarquable de leur estat (...), translatées de latin en françois par Charles-Annibal Fabrot, Cologny, Pernet, 1615 ; Honoré BoucHE, La Chorographie ou Description de Provence et l'histoire chronologique du même pays, par le sieur Honoré Bouche, depuis l'établissement de son comté jusques aujourd'hui, Aix-en-Provence, David, 1664, 2 vol ; Antoine de RuFFI, Histoire de la ville de Marseille, contenant tout ce qui s'y est passé de plus mémorable depuis sa fondation (...), Marseille, Garcin, 1642.

91. Ibid., $\mathrm{f}^{\circ} 252, \mathrm{v}^{\circ}$. Cette lutte entre Hercule et les princes espagnols serait par ailleurs symbolisée par la sculpture de l'Homme aux quatre jambes encore visible dans la ville et que Rulman décrit comme une «statue de trois corps et de six jambes " (actuellement in situ, entre la rue de l'Aspic et l'impasse des Quatre-Jambes). Ce mythe récurrent dans la littérature nîmoise a par ailleurs été étudié par Robert SAUZET, "L'image de Nîmes antique dans l'historiographie et la mémoire collective locale aux XVII ${ }^{\mathrm{e}}$ et XVIII ${ }^{\mathrm{e}}$ siècles ", in Claude Petitfrère, dir., Images et imaginaires dans la ville à l'époque moderne, Tours, Presses universitaires François-Rabelais, 1998, p. 49-61 et par Philippe CHAREYRE, « Mythes urbains et légendes villageoises. L'écriture de la fondation de Nîmes et de son territoire à la Renaissance (1533-1627) », in Véronique Lamazou-Duplan, dir., Ab urbe condita. Fonder et refonder la ville. Récits et représentations, Pau, Presses universitaires de Pau et des Pays de l'Adour, 2011, p. 163-188.

92. BCAN, ms 180, t. 1, f. $252 \mathrm{v}^{\circ}$. Voir à ce sujet l'ensemble du récit sur La fondation de l'ancien Nismes. 


\section{ABSTRACTS}

Thanks to its prestigious and antique past, Southern France appears to be a fruitful field of study to grasp antiquarianism during modern times. Based on the manuscripts of the Nîmois Anne de Rulman (1582-1632), this article depicts the context of archaeological discoveries, which are numerous in Nîmes at the begining of the seventeenth century. It also enlightens different types of protagonists, within various involvements, thus defining practices that surround the constitution of antiquities collections. Beyond that, Rulman's text reveals the development of a southern identity, shared by an antiquarian elite which gather around a common past - the Narbonese Gaul - a southern identity which however fades away face to local issues.

De par son prestigieux passé antique, le Midi de la France constitue un terrain d'études fécond pour l'appréhension de la curiosité antiquaire à l'époque moderne. En se fondant sur les manuscrits du Nîmois Anne de Rulman (1582-1632), l'article rend compte du contexte des découvertes archéologiques nîmoises, nombreuses en ce début du XVII siècle, de ses acteurs aux implications multiples, et ainsi des pratiques qui jalonnent la constitution des collections d'antiquités. Au-delà, le texte de Rulman laisse apercevoir l'élaboration d'une identité méridionale partagée par une élite antiquaire qui se reconnaît dans un passé commun - la Gaule Narbonnaise - une identité méridionale qui s'efface cependant face à des enjeux plus locaux.

\section{INDEX}

Mots-clés: Antiquaire, Collection d'antiquités, Anne de Rulman, Midi de la France, XVIIe siècle Keywords: Antiquarianism, Antiquities collection, Southern France, 17th century

\section{AUTHOR}

\section{MARIANNE FREYSSINET}

CRISES (EA 4424), Université Paul-Valéry, Montpellier mariannefreyssinet@gmail.com 